\title{
EARLY PRESENTATION OF NEUROFIBROMATOSIS TYPE 1
}

\author{
T. D.Thimmappa ${ }^{1}$, K.S.Gangadhar ${ }^{2}$, Harshavardhan Kushal Shetty ${ }^{3}$.
}

1. Associate Professor and Head of the Department, Department of ENT, Shimoga Institute of Medical Sciences, Government Mc. Gann Teaching Hospital, Shimoga.

2. Professor \& Dean, Department of Otorhinolaryngology, Shimoga Institute of Medical Sciences, Shimoga.

3. House Surgeon, Department of Otorhinolaryngology, Shimoga Institute of Medical Sciences, Shimoga.

\section{CORRESPONDING AUTHOR}

\author{
Dr. T. D. Thimmappa,
}

Department of ENT,

SIMS, Shimoga.

Email-drtdthimmappa@yahoo.com

\section{HOW TO CITE THIS ARTICLE:}

T. D. Thimmappa, K.S. Gangadhar, Harshavardhan Kushal Shetty. "Early presentation of neurofibromatosis type 1 in a 11 year old child". Journal of Evolution of Medical and Dental Sciences 2013; Vol2, Issue 26, July 1; Page: $4672-4675$.

ABSTRACT: Neurofibromatosis is one of the common genetic disorders affecting man. Incidence being 1 in 3,000. Family history is almost always present. Usually, the symptoms are seen in early childhood. However, an early presentation of NF1 presents with features which later develop into features seen in later adulthood. Swellings at atypical sites are sometimes misleading. However, correlation with other clinical findings helps to come to a diagnosis. Associated ocular, cutaneous, orthopaedic and central nervous system manifestations should be searched for. Significant family history should be extracted. The generalized form is easily diagnosed. But smaller lesions at sites such as the axilla and groin are ignored by the patient and clinically missed by the clinician. Here we discuss a case of Neurofibromatosis type 1 .

INTRODUCTION: Neurofibromatosis is a disorder of neural crest cells primarily affecting the growth of neural tissues. Cutaneous manifestations (commonly seen in NF type 1) consists of multiple café-au-lait spots and advanced disease with tumor (neurofibroma) arising from the nerve sheath. Cutaneous and CNS manifestations are associated with ocular as well as orthopaedic manifestations in varying degrees of severity.

CASE REPORT: A 11 year old female patient with the chief complaint of a swelling in the inner side of the left medial canthus since 3 years (fig.1). General physical examination revealed multiple hyperpigmented patches all over the body including axilla (fig2) and inguinal region, small nodules in the lower part of the abdomen and back fig 3). A neuro fibromatous nodule in the sole of the right foot fig 4) was found. Fullness in the left medial canthal region of the left eye. Family history revealed similar complaints in the father and the patient's elder brother. No similar complaints in the younger male sibling.

Laboratory evaluation was within normal limits. Investigative workup further confirmed the findings suggestive of Neurofibromatosis: Ophthalmoscopic examination revealed multiple mammillations in the iris of both eyes. X-ray did not reveal any bony involvement. CT scan of the 
PNS (coronal cut) revealed a soft tissue mass measuring approximately $2 \times 1.5 \mathrm{~cm}$ in the left medial canthal region without underling bony destruction.

DISCUSSION: $(1,2,3,4)$ This case exhibited findings consistent with neurofibromatosis type1.

Neurofibromatosis is a multisystem autosomal dominant genetic disorder occurring due to mutation in the long arm of chromosome 17 (NF1) of chromosome 21 (NF2). Neurofibromatosis type 1 is associated more with cutaneous manifestations (Multiple Café-au-lait spots) while Neurofibromatosis type 2 (bilateral acoustic neuroma) is associated more with CNS manifestations. The mutation is a deletion of the NF1 gene on chromosome 17 which codes for the neurofibromin 1 gene which serves as a tumor suppressor. Decreased production of this gene product leads to the range of clinical features.

Epidemiology: Internationally 1 in 3000-40001.

In patients of Asian origin, the prevalence is $1.81 / 1000$ and $0.95 / 1000$ in males and females respectively. (6) In India it is about roughly $0.3 / 1000$.

SEX: Males and females are affected equally.

AGE: Although the genetic change causing NF1 is present at conception, clinical manifestations appear slowly over many years.

Diagnosis often is made earlier in children born to an NF1-affected parent.

If an at-risk individual reaches the age of 10 years without meeting the diagnostic criteria for NF1, he or she is unlikely to be affected.

Classification of Neurofibromatosis according to Riccardi(1):

NF1 - Von Recklinghausen

NF2 - Acoustic neuroma

NF3 - Mixed (multiple brain and spinal tumors with CALM and neurofibroma)

NF4 - Variant (Both neurofibroma and CALM are present)

NF5 - Segmental (non-familial, CALM and/or neurofibroma limited to a unilateral segment)

NF6 - CALM (multiple CALM without neurofibroma)

NF7 - Late onset (Manifestations after 20 years of age, CALM may be absent)

NF8 - Not otherwise specified

DIAGNOSTIC CRITERIA: (5)

Two or more of the features listed if present establishes the diagnosis of NF1

1. 6 or more café au lait macules

- larger than $5 \mathrm{~mm}$ in greatest diameter in prepubertal individuals

- larger than $15 \mathrm{~mm}$ in greatest diameter in postpubertal individuals

2. Freckling in the axillary or inguinal regions

3. Optic pathway glioma

4. Two or more Lisch nodules

5. A distinctive osseous lesion, such as sphenoid dysplasia or thinning of the long bone cortex, with or without pseudoarthrosis

6. A first-degree relative with NF-1 according to the above criteria

7. Two or more neurofibromas of any type or 1 plexiform neurofibroma. 
In this patient 5 out of 7 criteria are fulfilled. However, in the presence of a positive family history just one more criteria need to be fulfilled.

Associated ocular manifestations in this patient are bilateral iris hamartomas (mamillations) which are again consistent with NF1. These mamillations develop into Lisch nodules with the advancement of age.

Due to the proximity of the swelling to the eye and orbit it is advisable to leave it untouched to avoid injury to surrounding structures.

Neurofibromatosis is a medical and surgical conundrum i.e, it is a puzzle that cannot be solved.

\section{REFERENCES:}

1. IADVL III edition Pg. 133.

2. Kanski textbook of ophthalmology.

3. Riccardi VM. Neurofibromatosis: clinical heterogeneity. Curr Probl Cancer;7:1-34

4. Bailley and Love's Textbook of Surgical Short cases.

5. Giulia Libero and Claudia Pizzo: A description of Neurofibromatosis type 1.

6. J Med Genet 1994;31:853-857 Neurofibromatosis type 1 in Israel: survey of young adults Ben Zion Garty, Arie Laor, Yehuda L Danon

Concluding the diagnosis as early presentation of Neurofibromatosis type 1

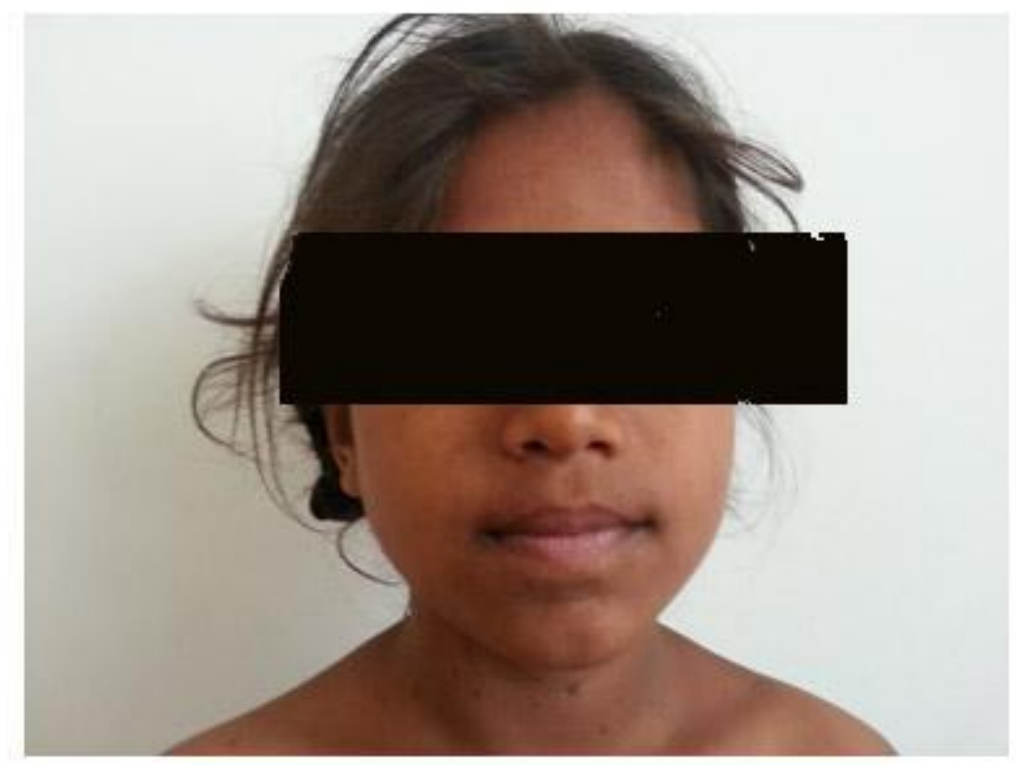




\section{CASE REPORT}

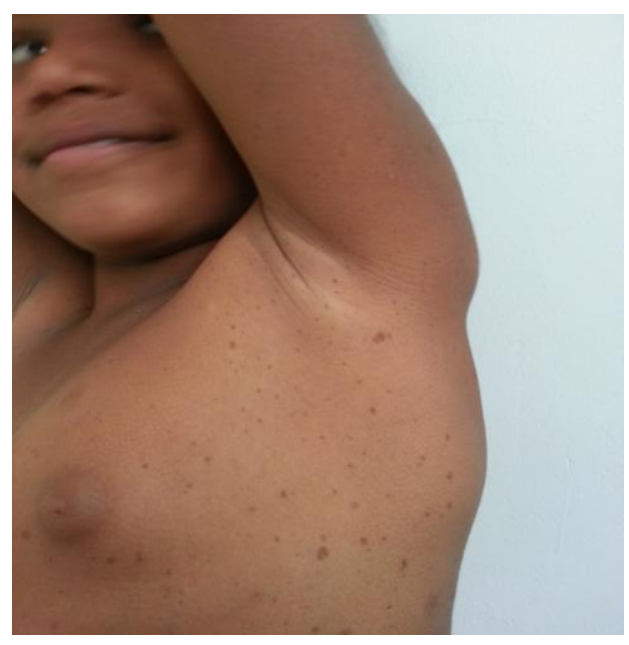

FIG2

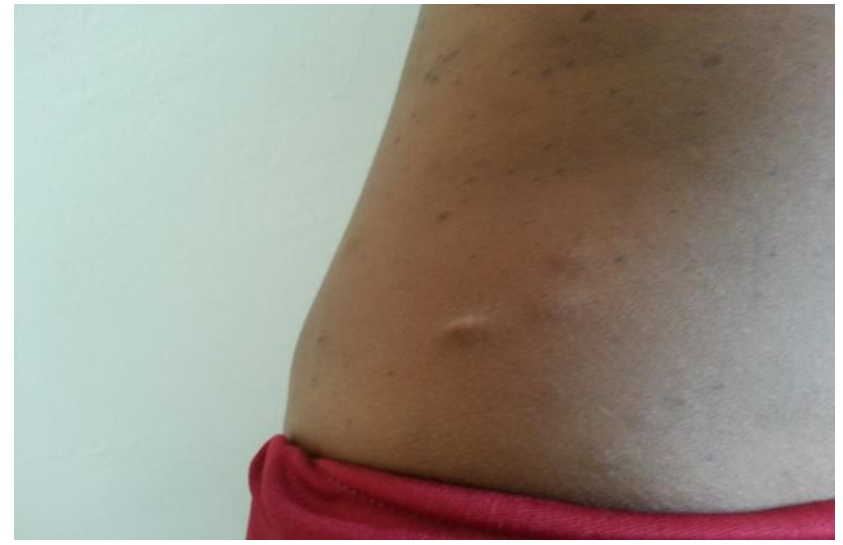

FIG 3

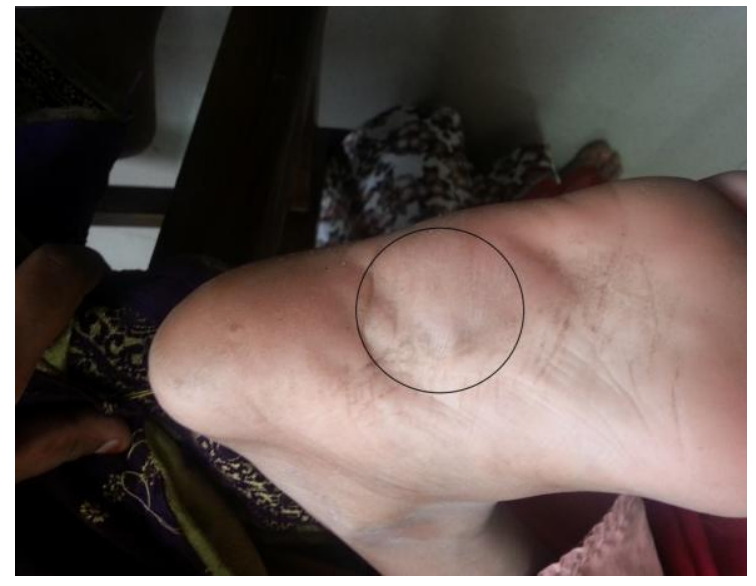

FIG 4: Neuro fibromatous Swelling over the plantar aspect of the right foot. 